\title{
The S.-T. Yau Center at National Chiao-Tung University
}

\section{by Song-Sun Lin and Wen-Wei Lin}

The Center's 3D Imaging Group was led by Professor Wen-Wei Lin and supervised by Prof. S. T. Yau. With the development of 3D geometric acquisition technologies, there is a massive amount of 3D geometric data available today. It is a great challenge to process and analyze 3D geometric data efficiently and accurately. Computational conformal geometry is an emerging interdisciplinary field, combining modern geometry theories from pure mathematics with computational algorithms from computer science and offering rigorous and practical tools for tackling massive geometric data processing problems.

From a theoretical point of view, computational conformal geometry has deep roots in mathematics and physics. In mathematics, it is at the intersection of many fields, such as algebraic topology, differential geometry, Riemann surface, harmonic analysis, and so on. It also has close relations to many fields of physics, such as the electromagnet field in electro- dynamics, elasticity deformation in mechanics, heat diffusion in thermal dynamics, modular space theory in super string theory. From a practical point of view, computational conformal geometry offers many powerful tools that handle a broad range of geometric problems in the engineering world.

The Computational Complexity Group, led by Prof. Ker-I Ko in Computer Science Department, joined the center in 2013. Computational complexity group studies computational complexity theory and its applications in various areas in theoretical computer science and mathematics. The theoretical study focuses on approximation and randomized algorithms using newly developed mathematical tools such as expander graphs and semidefinite programming. The applications include computability and complexity in real and complex analysis, computational game theory, computational learning theory, cryptography and computational biology.
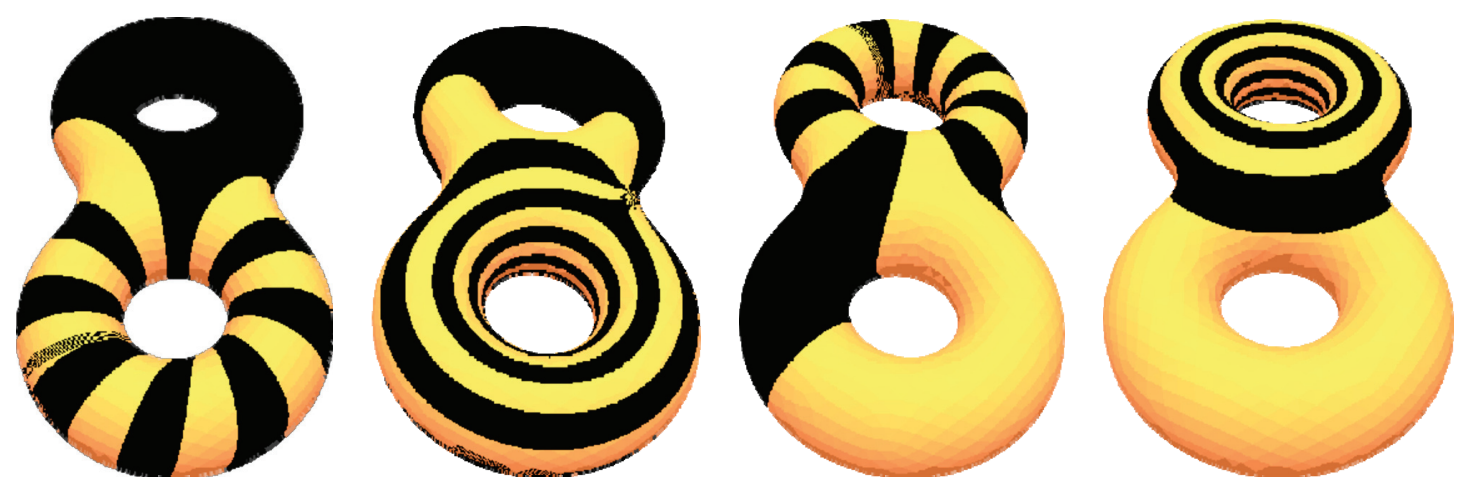

A holomorphic 1-form 

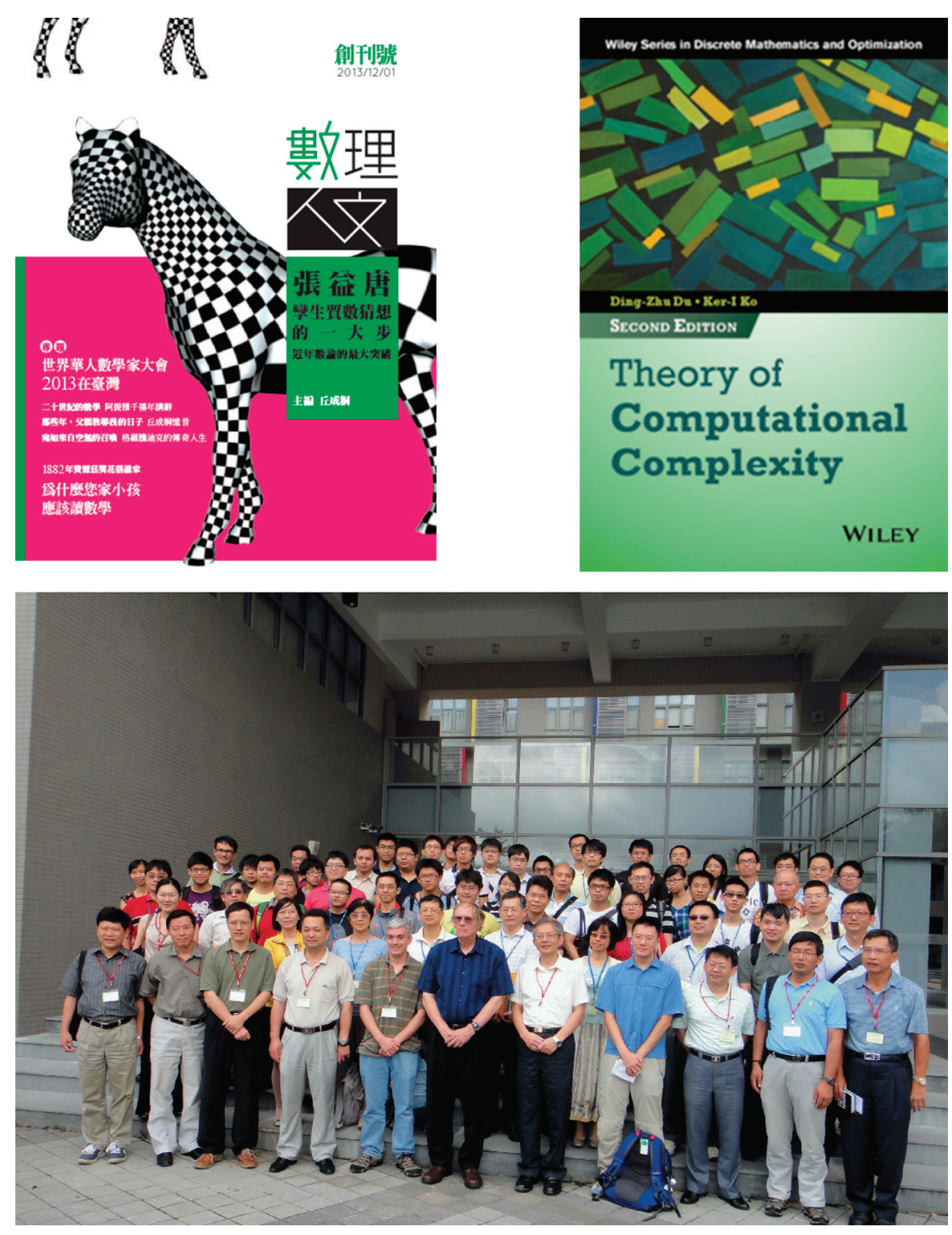

\section{Activity Highlights in 2013}

- Inducted two leading combinatorial mathematicians Fan Chung Graham and Ronald Graham as our university's chair professors and invited them to be the plenary speakers for 2013 International Conference on Combinatorics on July 11-12, 2013.

- Sponsored ICCM 2013 on July 14-19, 2013, Taipei; sponsored the 3rd India-Taiwan Confer- ence on Discrete Mathematics on Nov. 19-22, 2013; Co-organized 2013 NCTS Workshop on Numerical Linear Algebra and High Performance Computing (2013 NLA-HPC) on Dec. 9-12, 2013.

- Edited and issued "Mathematics, Science, History, and Culture Magazine" in July and December, 2013.

2013 International Conference on Combinatorics on July 11-12, 2013. 